\title{
Gastric Remnant Perforation after Roux-en-Y Gastric Bypass: A Case Report and Literature Review
}

\author{
Carlota Tuero $^{1} \oplus$, Gorka Docio $^{2}$, Victor Valenti ${ }^{3}$, Alicia Artajona ${ }^{4}$, Soledad Monton ${ }^{5}$
}

\begin{abstract}
Aim and objective: The aim and objective of this article was to focus on long-term complications after bariatric surgery, which are usually managed by general surgeons in the emergency department.

Background: Roux-en-Y gastric bypass (RYGBP) is one of the most commonly performed bariatric techniques in the world. Gastric remnant complications after this procedure are infrequent and poorly known. Furthermore, the diagnosis of this pathology may be challenging. Case description: We present the case of a 54-year-old woman with intense epigastric pain and history of uncomplicated laparoscopic RYGBP 18 years ago. After clinical, laboratory, and radiological examinations, the patient was diagnosed with a gastric remnant perforation. Laparoscopic surgery was performed, and the perforation was successfully repaired with primary suture and omental patch.

Conclusion: Gastric remnant perforation after bariatric surgery is not frequent and usually appears several years after the procedure. This type of pathology is presented without specific clinical manifestations and with few analytical alterations. Complementary radiological studies, such as computed tomography (CT) scan, should be performed. However, pneumoperitoneum and extravasation of oral contrast are usually absent. Depending on the size of the defect, primary suture or gastric remnant resection may be performed. Nevertheless, surgical treatment should not be delayed.

Clinical significance: Long-term complications after bariatric surgery are in many circumstances managed by general practitioner surgeons. The low incidence and scarce manifestations make the diagnosis of this pathology challenging. Furthermore, bariatric surgery is progressively increasing its presence all over the world. Complications after this procedure must be known and kept in mind because an early diagnosis is crucial to give a proper treatment and reduce morbidity and mortality.

Keywords: Bariatric surgery complications, Emergency surgery, Gastric bypass, Gastric remnant perforation, Pyloric perforation.

World Journal of Laparoscopic Surgery (2021): 10.5005/jp-journals-10033-1458
\end{abstract}

\section{BACKGROUND}

Roux-en-Y gastric bypass (RYGBP) is the gold standard treatment for morbid obesity achieving long-term weight loss and comorbidity resolution. Complications after this type of surgery must be known, especially long-term ones that sometimes are managed by general practitioner doctors.

Peptic ulcer disease is not frequent after RYGBP. Diagnosis is usually made after developing complications such as bleeding or perforation. However, literature is scarce, with 18 case reports and 5 case series. ${ }^{1}$ The main clinical symptoms are unspecific with epigastric abdominal pain associating with nausea and vomiting.

Gastric remnant perforation is a rare entity that should not be overlooked when exploring any patient with abdominal pain and history of bariatric surgery. It is essential to take this pathology into consideration in the differential diagnosis because altered anatomy may change the clinical presentation and radiological findings in bariatric patients. An early diagnosis and a proper treatment are crucial.

\section{Case Description}

A 54-year-old woman with a history of uncomplicated laparoscopic RYGBP and intense abdominal pain 18 years ago was admitted in the emergency department. She complained of epigastric pain for about 1 week, which had progressed in the last few days, radiating to the back. She also developed symptoms such as anorexia, nausea, and dizziness. Recent history was negative for smoking or alcohol abuse, but she claimed to have taken several doses of ibuprofen for lumbar pain.
1,2,4,5 Department of Surgery, Hospital Garcia Orcoyen, Estella, Navarra, Spain

${ }^{3}$ Department of Surgery, Clinica Universidad de Navarra, Pamplona, Navarra, Spain

Corresponding Author: Carlota Tuero, Department of Surgery, Hospital Garcia Orcoyen, Estella, Navarra, Spain, e-mail: ctuero@unav.es

How to cite this article: Tuero C, Docio G, Valenti V, et al. Gastric Remnant Perforation after Roux-en-Y Gastric Bypass: A Case Report and Literature Review. World J Lap Surg 2021;14(2):141-143.

Source of support: Nil

Conflict of interest: None

On physical examination, the patient was afebrile, without signs of sepsis. She had right upper quadrant and epigastric tenderness with deep palpation without rebound or percussion tenderness. Abdominal auscultation was normal. Her body mass index was $29 \mathrm{~kg} / \mathrm{m}^{2}$.

Laboratory examination showed anemia and elevated lipase and amylase activities. C-reactive protein level and white blood cell count were within normal limits.

No free air was seen on the abdominal X-ray, so a computed tomography $(\mathrm{CT})$ with intravenous and oral contrast was performed. Findings were correlative with acute pancreatitis, so the patient was admitted for observation. On clinical revaluation and despite medical treatment, 48 hours after admission, abdominal pain 
increased and laboratory findings worsened. The CT scan was repeated, and a prominent thickness of the anterior gastric antrum with fat stranding, a small amount of free fluid on right paracolic gutter, and a millimetric bubble of free air were observed (Fig. 1). For the diagnosis of perforated gastric remnant, the patient was taken to the operating room.

Laparoscopic surgery was performed where a 1-cm prepyloric perforation in the gastric anterior border with purulent ascites was found (Fig. 2). Cultures were taken, and the defect was closed with nonabsorbable barbed suture. Helicobacter pylori stool antigen test was negative. Postoperative course was uneventful, and she was discharged a week after the proton-pump inhibitor (PPI) treatment.

\section{Discussion}

Roux-en-Y gastric bypass is an effective technique achieving long-term weight loss reduction and preventing obesity-related comorbidities with reasonably low complication rates.

Gastric remnant complications after RYGBP, such as ischemia, perforation, bleeding, or neoplasia, are unusual. These complications have been described as late as 20 years after RYGBP, so the long-term follow-up is important in these patients.,

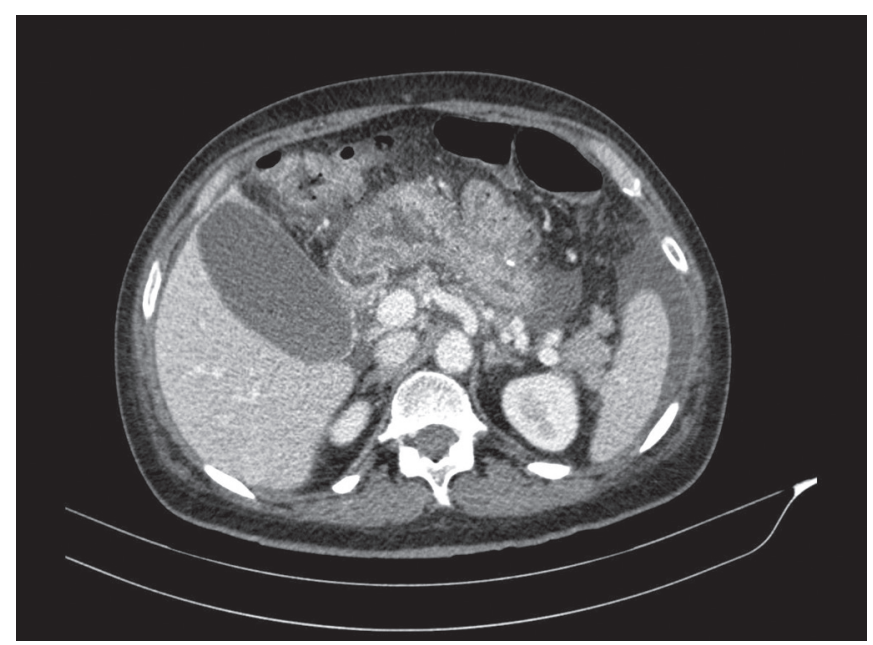

Fig. 1: CT scan: Thickness of the anterior gastric antrum, fat stranding, small amount of free perisplenic fluid, and a millimetric bubble of free air

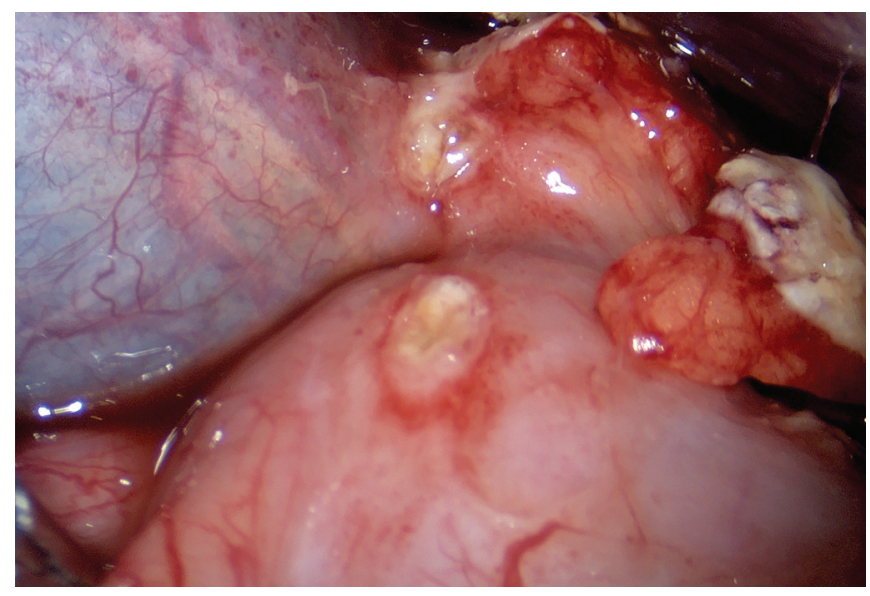

Fig. 2: Laparoscopic findings: 1-cm prepyloric perforation in the gastric anterior border
Risk factors for perforation of the gastric remnant are almost the same as those for marginal gastrojejunal ulcers. Mucosal ulcerations may be caused by excessive alcohol consumption, smoking, or nonsteroidal anti-inflammatory drug intake. ${ }^{3-5}$ Gastrin levels are reduced after RYGBP. However, the bypassed gastric segment still maintains its secreting capacities that vary depending on the gastric section hight. 'Besides, the acid produced in the bypassed stomach is not neutralized by the ingested food or washed out by gastric peristalsis, unlike what happens with normal anatomy. ${ }^{6}$ Moreover, the reflux of bile and the delayed bicarbonate secretion may also damage the mucosa. ${ }^{4,5,7}$ This chronic inflammatory stimulus may cause gastritis, metaplasia, and dysplasia.

$H$. pylori is also clearly associated with the formation of gastric ulcers with a prevalence of up to $85 \%$ in obese patients. ${ }^{5,8,9}$ International guidelines differ in their recommendations regarding the management of this infection. Nevertheless, considering $H$. pylori as a risk factor for gastric cancer and the difficult access to the excluded stomach after RYGBP, prior to surgery, bariatric patients should undergo a routine upper endoscopy in order to diagnose and treat this infection.

In postsurgery patients, urea breath tests are not reliable because there is no direct connection between the excluded stomach and the urea. ${ }^{10}$ False negatives may be found unless there is an infection on the gastric pouch, so stool antigen detection is probably the best noninvasive diagnostic method with a sensitivity and specificity of over $90 \%{ }^{1,10}$ However, histological confirmation is the gold standard diagnostic technique. If there is an H. pylori infection on the bypassed stomach, eradication therapy is mandatory. Nevertheless, there are scarce data regarding treatment success rates in these patients.

Regarding the diagnosis of gastric remnant perforation, epigastric pain is the most frequent symptom, which is sometimes associated with signs of sepsis. Free air in abdominal radiography is rare because the excluded stomach does not contain intraluminal air. ${ }^{1}$ Therefore, negative radiological findings should not exclude the diagnosis of this entity. Furthermore, if free air is present in the radiography, a gastrogastric fistula or a jejunojejunostomy obstruction should be suspected..$^{11}$ CT scan with oral contrast is the main diagnostic method when a perforated ulcer is suspected in a patient after RYGB. The most common finding in the CT is free peritoneal fluid with an inflammatory process in the right upper quadrant. Conversely, extravasation of oral contrast and/or pneumoperitoneum are seldom-observed. ${ }^{3,5}$

Gastric cancer underlying the ulceration is uncommon but must also be discarded. Ulceration may be a manifestation of this entity. Therefore, histological confirmation and diagnostic visualization are mandatory. In case of gastric cancer confirmation, treatment does not differ from management in patients with prior partial gastrectomy:gastric remnant gastrectomy with D2 lymphadenectomy. ${ }^{2}$

To access the bypassed gastroduodenal segment, upper endoscopy is useless, so different modalities have been described. It is possible to address directly to the excluded stomach with a percutaneous approach guided by ultrasound or CT. Furthermore, a temporary gastrostomy tube can be placed. Other options are retrograde gastroduodenoscopy with a pediatric colonofibroscope and double-balloon enteroscopy. Laparoscopic-assisted transgastric remnant endoscopy is another alternative. ${ }^{2,7,10}$

Surgical treatment of gastric remnant perforation is usually laparoscopic repair with primary defect closure, omental patch, and drainage. However, the scarce data do not allow to make any general 
recommendations. If there is an acute perforation with a small defect, the closure after biopsy is usually easy, performing a safe and short laparoscopic procedure. Besides, it is recommendable to enable a gastric remnant access in the same surgery. It is also recommendable to perform a selective vagotomy to reduce the acid production. ${ }^{1}$ Gastric resection is sometimes executed in the second stage or when there is a large perforation or ischemia of the gastric remnant. ${ }^{2}$ Resecting the excluded stomach may decrease the gastric acid production and avoid the formation of gastrogastric fistula. ${ }^{3}$ However, resecting the stomach implies shortterm complications such as bleeding of omental vessels, necrosis of omental fat with abscess formation, or duodenal stump leakage. Other complications such as bacterial overgrowth and vitamin B12 deficiency have also been described. ${ }^{3,10}$

Regarding discharge and follow-up, guidelines recommend H. pylori eradication when tests were positive, and recommend 1-month treatment with PPI., ${ }^{12}$

\section{Conclusion}

Bariatric and general surgeons managing RYGBP patients should not overlook gastric remnant complications in their differential diagnosis of abdominal pain. Gastric remnant perforation should be taken into account if a bariatric patient presents with severe, epigastric abdominal pain without important radiological findings. Risk factors are not clear, but $H$. pylori eradication is recommended. Laparoscopic exploration should be performed urgently because the operation time interval once the perforation has occurred is an important predictor for morbidity and mortality. Defect closure or gastric remnant resection may be performed without any general recommendations, but biopsies should be taken.

\section{OrCID}

Carlota Tuero (ㄴ) https://orcid.org/0000-0001-8577-5603

\section{References}

1. Plitzko G, Schmutz G, Kröll D, et al. Ulcer disease in the excluded segments after Roux-en-Y gastric bypass: a current review of the literature. Obes Surg 2020;31(3):1280-1289. DOI: 10.1007/s11695-02005123-w.
2. Iranmanesh P, Manisundaran NV, Bajwa KS, et al. Management of acute gastric remnant complications after Roux-en-Y gastric bypass: a single-center case series. Obes Surg 2020;30(7):2637-2641. DOI: 10.1007/s11695-020-04537-w. Available from: https://pubmed.ncbi. nlm.nih.gov/32162207/.

3. Iskandar ME, Chory FM, Goodman ER, et al. Diagnosis and management of perforated duodenal ulcers following Roux-En-Y gastric bypass: a report of two cases and a review of the literature. Case Rep Surg 2015;2015:1-4. DOI: 10.1155/2015/353468. Available from: https://pubmed.ncbi.nlm.nih. gov/pmc/articles/PMC4408622/?report=abstract.

4. Ovaere S, Tse WHW, Schipper EE, et al. Perforation of the gastric remnant in a patient post-Roux-en- $Y$ gastric bypass. BMJ Case Rep [Internet]. 2016;2016. DOI: 10.1136/bcr-2015-213862. Available from: https://pubmed.ncbi.nlm.nih.gov/pmc/articles/ PMC4785498/?report=abstract.

5. Dai L, Shah MM, Rosenblatt S. Perforated gastric remnant ulcer after laparoscopic gastric bypass. BMJ Case Rep 2018;2018. DOI: 10.1136/ bcr-2017-222190. Available from: https://pubmed.ncbi.nlm.nih. gov/29622702/.

6. Bjorkman DJ, Alexander JR, Simons MA. Perforated duodenal ulcer after gastric bypass surgery. Am J Gastroenterol 1989;84(2):170-172.

7. Tornese S, Aiolfi A, Bonitta G, et al. Remnant gastric cancer after Roux-en-Y gastric bypass: narrative review of the literature. Obes Surg 2019;29(8):2609-2613. DOI: 10.1007/s11695-019-03892-7. Available from: https://pubmed.ncbi.nlm.nih.gov/31001760/.

8. Cleator LGM, Rae A, Birmingham CL, et al. Ulcerogenesis following gastric procedures for obesity Obes Surg 1996;6(3):260-261. DOI: $10.1381 / 096089296765556881$. Available from: https://pubmed. ncbi.nlm.nih.gov/10729870/.

9. Csendes A, Burgos AM, Smok G, et al. Endoscopic and histologic findings of the foregut in 426 patients with morbid obesity. Obes Surg 2007;17(1):28-34. DOI: 10.1007/s11695-007-9002-9. Available from: https://pubmed.ncbi.nlm.nih.gov/17355765/.

10. Gypen BJ, Hubens GJA, Hartman V, et al. Perforated duodenal ulcer after laparoscopic gastric bypass. Obes Surg 2008;18(12):1644-1646. DOI: 10.1007/s11695-008-9530-y. Available from: https://pubmed. ncbi.nlm.nih.gov/18443886/.

11. Pohl D, Schmutz G, Plitzko G, et al. Perforated duodenal ulcers after Roux-Y Gastric Bypass. Am J Emerg Med 2018;36(8):1525.e1-1525.e3. DOI: 10.1016/j.ajem.2018.04.057. Available from: https://pubmed. ncbi.nlm.nih.gov/29716802/.

12. Satoh K, Yoshino J, Akamatsu T, et al. Evidence-based clinical practice guidelines for peptic ulcer disease. J Gastroenterol 2016;51(3):177-194. DOI: 10.1007/s00535-016-1166-4. Available from: https://pubmed. ncbi.nlm.nih.gov/26879862/. 